\title{
Phase separation in the two-dimensional electron liquid in MOSFETs.
}

\author{
B.Spivak \\ Physics Department, University of Washington, Seattle, WA 98195
}

\begin{abstract}
We show that the existence of an intermediate phase between the Fermi liquid and the Wigner crystal phases is a generic property of the two-dimensional pure electron liquid in MOSFET's at zero temperature. The physical reason for the existence of these phases is a partial separation of the uniform phases. We discuss properties of these phases and a possible explanation of experimental results on transport properties of low density electron gas in Si MOSFET's. We also argue that in certain range of parameters the partial phase separation corresponds to a supersolid phase discussed in $[17]$.
\end{abstract}

\section{INTRODUCTION}

This work is motivated by experiments [1-13] on transport properties of the two dimensional electron system in high mobility Si-MOSFET's at small electron concentration $n$. These experiments raised doubts about the applicability of the Fermi liquid theory and the conventional theory of localization $[14,15]$ to the two dimensional disordered electron liquid at low temperatures. The aim of this article is to prove the existence of zero temperature phases of the two dimensional pure electron liquid in MOSFET's which are intermediate between the Fermi liquid and the Wigner crystal. These phases exist in some interval of concentrations $n_{W}<n<n_{L}$. The values of the critical concentrations $n_{W}$ and $n_{L}$ are estimated below.

This phenomenon is due to a tendency for phase separation which originates from the existence of a first-order phase transition between the Fermi liquid and the Wigner crystal phases as a function of $n$. The difference between the crystal-liquid phase transition in MOSFET's and the usual first order phase transitions in neutral systems is the following. In neutral systems with first order phase transitions the energy of the surface between the phases is positive and the minimum of the free energy corresponds to a minimal surface area and to a global phase separation. In charged systems, like electrons on a positive frozen background, global phase separation does not occur because of a large Coulomb energy associated with a non-uniform distribution of electron density. The electron liquid in MOSFET's, in a sense, is a system intermediate between these two limiting cases. Similarly to the neutral systems with first order phase transitions, the electron liquid in MOSFET's exhibits phase separation. On the other hand the surface energy of a minority phase droplet of a large enough radius turns out to be negative. As a result at different $n$ there is a variety of intermediate phases in this system which are different both from the Fermi liquid and from the Wigner crystal.

The electron system with phase separation demonstrates a number of unusual features. If $0<\left(n-n_{W}\right) \ll\left(n_{L}-n_{W}\right)$, the state of the system corresponds to a small concentration of Fermi liquid droplets embedded into the Wigner crystal. The main difference between such a state and the usual Wigner crystal is that it is not pinned by small disorder and can bypass obstacles, while the classical crystals at zero temperature are pinned by an infinitesimally small amount of disorder [16]. Phenomenologically this state of matter is similar to the supersolid phase proposed in [17] for the case of $\mathrm{He}^{3}$ and $\mathrm{He}^{4}$. The difference is that in our case the origin of droplets of liquid embedded in the crystal is classical electrostatic, whereas, in the case [17] the existence of vacancies and interstitials in the ground state of quantum crystals is of quantum origin.

If $0<\left(n_{L}-n\right) \ll\left(n_{L}-n_{W}\right)$, then the state of the system corresponds to a small concentration of Wigner crystal droplets embedded into the Fermi liquid. At small concentrations and small temperatures, in principle, these droplets can be considered as quasiparticles.

Droplets of a minority phase interact at large distances via short-range dipole forces rather than via Coulomb forces. This means that at $T=0$ and at small droplet concentration the system of such "droplet quasiparticles" should be in a liquid state similar to $H e^{3}$ and $H e^{4}$ which are also liquids at small densities. Thus we can describe the system by two-fluid hydrodynamics. However, the statistics of these quasiparticles remains unknown.

At zero temperature the one-dimensional boundary between the liquid and the solid is a quantum object itself. Due to zero-point oscillations of its position there is a region where the wave function has a form which is intermediate between the Fermi liquid and the Wigner crystal. Since the electron densities of the Wigner crystal and the Fermi liquid are slightly different the fluctuations of the position of the boundary is associated with the fact that the number of quasiparticles in the Fermi liquid is not conserved. 
On the mean-field level this picture of droplet formation in the electron liquid in MOSFET's is similar to the partial phase separations which occur in ferromagnetic films [18], charged polymers [19,20], neutron stars [21], doped manganites (see for example [22,23]), HTC superconductors [24-27] and 2-dimensional electron systems in the quantum Hall regime [28]. All these systems demonstrate a short-ranged tendency for phase separation which is thwarted by a long range Coulomb interaction preventing global phase separation.

The paper is organized as follows. In chapter 2 we show that there is an interval of electron concentrations in which the system is unstable with respect to the phase separation. We also estimate the size of minority phase droplets embedded into the majority phase and the temperature and magnetic field dependence of the droplet concentration. In chapter 3 we discuss transport properties of different nonuniform phases associated with the phase separation. In section 4 we compare the theoretical and experimental results on transport properties of the low density electron gas in Si MOSFET's.

\section{PHASE SEPARATION NEAR THE POINT OF THE FERMI-LIQUID-WIGNER CRYSTAL PHASE TRANSITION}

In this chapter we show that a partial phase separation is a generic property of pure 2-D electron liquids in MOSFET's. Consider a two-dimensional electron liquid of density $n$ in a MOSFET separated by a distance $d$ from a metallic gate. Electrons interact via Coulomb interaction while a global electric neutrality of the system is enforced by the metallic gate with a positive charge density $e n$. The energy density of the system per unit area $\epsilon(n)=\epsilon^{(C)}+\epsilon^{(e l)}$ is a sum of the energy density of the capacitor $\epsilon^{(C)}=(e n)^{2} / 2 C$ and the internal energy density of the electron liquid $\epsilon^{(e l)}$. In the case of a uniform electron distribution the capacity per unit area is $C=C_{0}=1 / d$.

At high electron densities $n a_{B}^{2} \gg 1$ the kinetic energy of electrons is larger than the potential energy and the interaction can be taken into account by a perturbation theory. (Here $a_{B}$ is the electron Bohr radius.) In this case the system can be described by Fermi liquid theory, the difference between the effective $m^{*}$ and the bare $m$ electron masses is small, and $\epsilon^{(e l)}=\epsilon_{L}^{(e l)} \sim n^{2} / m$. On the other hand, in the opposite limit $n a_{B}^{2} \ll 1$ (but still $n d^{2} \gg 1$ ) the potential Coulomb energy of electrons is much larger than the kinetic energy and the ground state of the system is a Wigner crystal with $\epsilon^{(e l)}=\epsilon_{W}^{(e l)}=-e^{2} n^{3 / 2}$ (see, for example, [29]). Thus at zero temperature there is a critical electron concentration $n_{c}$ where the phase transition between the Fermi liquid and the Wigner crystal phases takes place. According to Landau mean field theory this transition is of the first order (see for example [30]). The $n$-dependence of the energy densities of the Fermi liquid $\epsilon_{L}(n)=\epsilon^{C}+\epsilon_{L}^{(e l)}$ and the Wigner crystal $\epsilon_{W}(n)=\epsilon^{(C)}+\epsilon_{W}^{(e l)}$ phases near the critical density $n_{c}$ is shown schematically in Fig.1a.

In the limit of small densities $n d^{2} \ll 1$, due to the existence of the image charges in the gate, the interaction between adjacent electrons has a dipole character. In this case the ratio between the potential and the kinetic energy decreases as $n$ decreases. Therefore, the small electron $n$ the electron system is a weakly interacting Fermi liquid. Thus we arrive at the conclusion that there exists another critical point $n_{c}^{(1)} \sim 1 / d^{2}$ which corresponds to a second Wigner crystal-Fermi liquid transition. The phase diagram of the electron system at $T=0$ is shown in Fig.1b. If $d<d^{*} \sim 38 a_{B}$, than the system is in the liquid state at any value of $n$. Here the factor 38 is the result of numerical simulations [31].

\section{A. The mean field description of the phase separation.}

In the approximation when $C=C_{0}$ the qualitative picture of the phase transition is the same as the picture of any first order phase transition in neutral systems. In particular, there is an interval of electron densities $n_{W}<n<n_{L}$ shown in the Fig.1a where there is a phase separation, which means that there is a spatially nonuniform distribution of the Wigner crystal and Fermi liquid phases coexisting in equilibrium. In the case of large $d$ one can linearize $\epsilon_{L, W}^{(e l)}(n)$ near the point $n=n_{c}$. As a result, we have

$$
n_{L, W}=n_{c} \pm \frac{\left(\mu_{W}-\mu_{L}\right)}{2 e^{2} d}
$$

where $\mu_{W, L}=\left.\left(d \epsilon_{W, L}^{(e l)} / d n\right)\right|_{n=n_{c}}$.

One can get from Eq.1 an estimate $n_{c} a_{B} / d$ for the size of the interval of electron densities where the phase separation occurs. Values of $d / a_{B}$ in various MOSFET's range from of order one to 50. 
The relative fractions of these phases $x_{W}$ and $x_{L}$ are determined by the Maxwell rule. At $\left(n_{L}-n\right) \ll\left(n_{L}-n_{W}\right)$ the fraction of the area occupied by the Wigner crystal $x_{W} \ll 1$ is small while in the case $\left(n-n_{W}\right) \ll\left(n_{L}-n_{W}\right)$ the fraction of the area occupied by the Fermi liquid $x_{L} \ll 1$ is small.

$$
x_{W, L}= \pm \frac{n-n_{W, L}}{n_{c}}
$$

The compressibility of the system $\nu=d^{2} \epsilon / d n^{2}$ should exhibit jumps of order $e^{2} d$ at points $n=n_{L}, n_{W}$.

The crucial difference between first order phase transitions in neutral systems and in the system of electrons in MOSFET's arises when one considers shapes of the minority phases. In the case of neutral systems the surface energy density $\sigma$ is positive. Therefore in equilibrium the system should have a minimal area of the surface separating the phases, leading to global phase separation. On the other hand, in the three dimensional charged systems the global phase separation is impossible because of the large Coulomb energy associated with the charge separation. It is possible, however, that in this case the electron system consists of bubbles and stripes of different electron density $[24,27]$, provided the tendency for phase separation is strong enough.

The situation in MOSFET's is very different. On one hand, in the approximation when $C=C_{0}$ global phase separation is possible at an arbitrary value of $\left(\mu_{W}-\mu_{L}\right)$. On the other hand, it turns out that for large droplets of the minority phase the surface energy is negative. To prove this one has to take into account the finite size corrections to the standard formula for the capacitance [32]

$$
C=C_{0}+\frac{R}{A} \ln \frac{16 \pi R}{d}
$$

where $A$ and $d$ are the capacitor area and thickness respectively and $R=\sqrt{A}$ is the capacitor size. Consider, for example, the case when $x_{W} \ll 1$. Then $x_{W}$ can be determined by the Maxwell rule in the approximation when the second term in Eq.3 is neglected and $C=C_{0}$. Expanding $\epsilon(n)$ with respect to the second term in Eq.3 and taking into account also the microscopic surface energy we have an expression for the energy of the surface

$$
E_{(\text {surf })}=-\frac{1}{2} N_{W} e^{2}\left(n_{W}-n_{L}\right)^{2} d^{2} R_{W} \ln \frac{16 \pi R_{W}}{d}+N_{W} \sigma 2 \pi R_{W}
$$

We assume that the Wigner crystal phase embedded into the liquid consists of droplets of radius $R_{W}$ and concentration $N_{W}$ and take into account that inside the droplet $n \sim n_{W}$. Thus, at large $R_{W}$ the surface energy Eq.4 turns out to be negative. We have to find a minimum of Eq.4 at a given total area occupied by the minority phase, which gives us the characteristic size of the droplet

$$
R_{W} \sim \frac{d}{16 \pi} e^{\gamma}
$$

with $\gamma=\left(e^{2} \sigma\right) / 2 \pi\left(\mu_{W}-\mu_{L}\right)^{2}$. A similar expression was obtained in [33] for a different problem.

The analogous calculation for the case $x_{L} \ll 1$ gives the expression for the radius of liquid droplets embedded into the crystal which is identical to Eq.5.

At the point of the transition the values of $\sigma$ and $\left(\mu_{W}-\mu_{L}\right)^{2} / e^{2}$ are of the same order and at present nothing is known about the value of the dimensionless parameter $\gamma$. Even the fact that $\sigma>0$ is not proven. I would like to also note that in the case of the first order phase transitions which are close to the second order one we always have $\gamma \ll 1$.

In this article we assume that $\gamma \geq 1$. To illustrate the physical meaning of this inequality we consider the case when 2-D electron liquid is compensated by a uniformly charged positive frozen background with a charge density $e n$. In this case the Coulomb energy of a droplet associated with the phase separation is, roughly, $R / d$ times larger than in the MOSFET's case. The most dangerous point with respect to the phase separation instability is $n=n_{c}$ (see Fig.1a). For example, let us compare the energies of the uniform liquid state with $n=n_{c}$ and a nonuniform state which contains two droplets embedded into the liquid. The first droplet is a liquid with electron concentration $n_{1}=n_{c}+\delta n$, while the second term is a crystal with electron concentration $n_{2}=n_{c}-\delta n$. Suppose the droplets have the same radius $R$. Linearizing $\epsilon_{L, W}(n)$ with respect to $\delta n$ we estimate the energy difference $\delta E$ between these two states as

$$
\delta E \sim\left(\mu_{L}-\mu_{W}\right) \pi R^{2} \delta n+\frac{\left(e \delta n \pi R^{2}\right)^{2}}{R}+2 \pi R \sigma
$$

The first term in Eq.6 corresponds to a decrease of the energy due to the phase separation. The second one corresponds to the positive Coulomb energy associated with the nonuniform distribution of the electron density and the third term is the surface energy. A minimization of Eq.6 with respect to $\delta n$ gives us $\delta n \sim\left(\mu_{W}-\mu_{L}\right) / R e^{2}$ and 


$$
\delta E_{\text {min }} \sim\left(2 \pi \sigma-\frac{\left(\mu_{W}-\mu_{L}\right)^{2}}{e^{2}}\right) R
$$

The assumption $\gamma>1$ means that $E_{\text {min }}$ in Eq.7 is positive and that 2-d electron liquid on a frozen positive background does not exhibit a phase separation. (We would like to metion that if the value of $\left|\mu_{W}-\mu_{L}\right|$ is big enough a microscopic phase separation in the charged liquid on frozen positive background can take place even in 3D case. Such situation has been considered in $[24-27,58]$ )

On the mean field level our problem is similar to $[18,19]$. Using this analogy we conclude that in the middle of the interval $\left(n_{W}, n_{L}\right)$ there is a stripe phase. The phase diagram of the system is shown schematically in Fig.2. The main difference with $[18,19]$ is the following. In $[18,19]$ all phase transitions between uniform, bubble and stripe phases are of the first order, whereas in our case the transitions between uniform (Fermi liquid and Wigner crystal) phases and the bubble phases are continuous. The transitions between the bubble phases and the stripe phase would be the first order one. However, such a transition would have an interval of concentrations where phase separation would take place. In this case the presented above arguments could be repeated. Thus we expect more complicated structures than bubbles and stripes phase to exist between the bubble and the stripe phases. Since the complete solution of this problem remains to be found we indicated this in Fig.2 by shaded lines.

Let us now estimate dependence of $x_{W, L}\left(T, H_{\|}\right)$on the temperature $T$ and the magnetic field $H_{\|}$parallel to the film. It is determined by the corresponding dependence of the free energies for the Fermi liquid and Wigner crystal phases. At small $T$ and $H_{\|}$one can neglect the $T$ and $H_{\|}$dependences of $\epsilon_{W, L}$ and we have the following expression for the free energy densities of the liquid and the Wigner crystal phases

$$
F_{W, L}\left(H_{\|}\right)=\epsilon_{W, L}-M_{W, L} H_{\|} n-T n S_{W, L}
$$

where $S_{W}$ and $S_{L}$ are the entropies of the crystal and the liquid phases respectively, while $M_{W}$ and $M_{L}$ are the corresponding spin magnetizations per electron.

As a result, one can obtain how $x_{W, L}\left(T, H_{\|}\right)$, and $n_{c W, L}\left(T, H_{\|}\right)$depend on $T$ and $H_{\|}$by making the following substitution in Eqs.1,2

$$
\left(\mu_{W}-\mu_{L}\right) \rightarrow\left(\mu_{W}-\mu_{L}\right)-\left(M_{W}-M_{L}\right) H_{\|}-T\left(S_{W}-S_{L}\right)
$$

At small $\mu_{B} H_{\|} \ll T \ll E_{F}$ we have $M_{W, L}=\chi_{W, L} H_{\|}$, where $\chi_{W}$ and $\chi_{L}$ are linear susceptibilities of the crystal and the liquid respectively. (Here $\mu_{B}$ is the Bohr magneton and $E_{F}$ is the Fermi energy.) At low temperature $T \ll E_{F}$ the spin susceptibility of the Wigner crystal $\chi_{W} \sim \mu_{B}^{2} / T \gg \chi_{L}$ is much larger than the spin susceptibility of the Fermi liquid. The entropy of the crystal $S_{W} \sim \ln 2 \gg S_{L} \sim T / E_{F}$ is mainly due to the spin degrees of freedom and much larger than the entropy of the Fermi liquid. Thus $x_{W}$ increases linearly with $T$ and quadratically with $H_{\|}$, which means that both the temperature and the magnetic field parallel to the film drive the electron system toward the crystallization [34]. (We assumed that the temperature is larger than the exchange energy between spins in the Wigner crystal). These effects are known in the physics of $\mathrm{He}^{3}$ as Pomeranchuk effects.

In the intermediate interval of magnetic fields $T<\mu_{B} H_{\|}<E_{F}$ spins in the Wigner crystal are completely polarized while the Fermi liquid is still in the linear regime. In this case $x_{W}$ increases linearly with $H_{\|}$.

At high magnetic field $H_{\|}>H_{\|}^{c} \sim E_{F} / \mu_{B}$ both Fermi liquid and Wigner crystal are spin polarized and $x_{W}\left(T, H_{\|}\right)$ saturates as a function of $H_{\|}$. We assume that $\epsilon_{L}\left(H_{\|}=0\right)<\epsilon_{L}\left(H>H_{\|}^{c}\right)$ and, therefore $x_{W}\left(H_{\|}=0\right)<x_{W}\left(H>H_{\|}^{c}\right)$. On the other hand, the spin entropy of the Wigner crystal is frozen in this case. As a result, at $H_{\|}>H_{\|}^{c}$ the temperature dependence of $x_{W, L}\left(T, H_{\|}\right)$is suppressed significantly.

\section{B. Quantum properties of the droplets of minority phase embedded into the majority one.}

In principle, at small enough concentrations and at small temperatures droplets of the minority phase embedded into the majority one should behave as quasiparticles. Since the system is translationally invariant, they should be characterized by momentum (or by quasi-momentum). The momentum coincides with the flux of mass. Thus these quasiparticles carry a mass $M^{*}$, a charge $e M^{*} / m$ and a spin. The characteristic temperature of quantum degeneracy is $T^{*} \sim N_{W} / M^{*}$.

The value of $M^{*}$ depends on the mechanism of motion of the droplets, which in turn depends on whether the surface between the crystal and the liquid is rough or smooth.

Consider for example the case of Wigner crystal droplet embedded into the liquid. In the case of a smooth surface, motion of the droplet is associated with a redistribution of the liquid mass on the distance of order $R_{W}$. In this case we can estimate the effective mass of the droplet as 


$$
M^{*} \sim m n_{c} \pi R_{W}^{2}
$$

In the case of rough surfaces the motion of the droplet is associated with melting and crystallization of different parts of it. Since the $\left(n_{L}-n_{W}\right) \ll n_{C}$ the liquid mass to be distributed and, consequently, effective mass of the droplet

$$
M^{*} \sim m\left(n_{L}-n_{W}\right) \pi R_{W}^{2}
$$

in this case is much smaller than Eq.10.

Droplets of the minority phase interact at large distances via short-range dipole forces rather than via Coulomb forces. At small enough concentration of the droplets the amplitude of quantum (or classical) fluctuations of their positions is larger than the typical distance between them. Thus the liquid droplets are distributed uniformly over the whole crystal. In other words, at $T=0$ the system of such "droplet quasiparticles" should be in a liquid state similar to $\mathrm{He}^{3}$ and $\mathrm{He}^{4}$ which are also liquids at small densities. Thus we can describe the system by two-fluid hydrodynamics. In this case statistics of the "droplet quasiparticles" becomes important. In this respect we would like to mention a difference between the droplets of the liquid embedded into the crystal and the droplets of the Wigner crystal embedded into the liquid.

a. The droplets of the liquid are topological objects which, in principle, are not different from vacancies or interstitials in quantum crystals $\mathrm{He}^{3}$ and $\mathrm{He}^{4}$. In order to create such objects in Wigner crystal one has to add or to remove from the lattice an integer number of electrons. Therefore, the liquid droplets have a definite statistics: they are either fermions or bosons [17].

The main feature of the phase where there are droplets of liquid embedded into the crystal (supersolid) is it's ability to bypass static obstacles. In other words, unlike conventional crystals supersolids are not pinned by disordered potential of small amplitude. This will manifest itself in the finite conductivity of the system.

From the phenomenological point of view this is very similar to the scenario of "supersolid" which has been introduced by A.F.Andreev and I.M.Lifshitz [17] for quantum crystals of helium near the quantum melting point. They assumed that the crystals contain zero point defects (vacancies or interstitials) in the ground state and therefore the number of atoms and number of sites in the crystals are different. The difference with [17] is that the origin of the negative surface energy Eq.4 is purely classical. Conversely, following [17] the existence of point defects in the ground state could be of quantum origin. Namely, the kinetic energy of the point defects can be larger than the energy required for their creation. Thus, the supersolid phase [17] can be considered as a particular case of a more general situation of the phase separation when the radius of liquid droplets embedded into the crystal is of order $n^{-2}$. This would mean that the surface energy is renormalized to a small (or negative) value. Indications of the existence of such a phase have been reported in numerical simulations [35].

b. The case of droplets of the Wigner crystal embedded into the Fermi liquid is different because they are not topological objects. In principle such droplets can contain an additional charge and spin which can be fractional or even irrational. A fundamental problem associated with this fact is that statistics of such quasiparticles is unknown.

To illustrate this point we consider a process of tunneling between two states: a state of uniform Fermi liquid and a state when there is one crystalline droplet embedded into the Fermi liquid. These two states have different total electron charge. Thus the tunneling between these states is associated with a redistribution of this charge to (and from) the infinity. It is important that the action $S$ associated with this process in the pure two dimensional case is finite. One can estimate it in a way similar to [36]. On distances larger than the droplet size $R_{W}$ one can write the action in terms of the time-dependent electron density $\tilde{n}(\mathbf{r}, t)$

$$
S \sim \int d t d \mathbf{r} \frac{[e \tilde{n}(\mathbf{r}, t)]^{2}}{C_{0}} \sim \int d t \frac{\left(e M^{*}\right)^{2} d}{m^{2}} \frac{1}{R^{2}(t)}
$$

Here $t$ is the imaginary time and $M^{*}$ is given by Eq.11. We approximate that $\tilde{n}(\mathbf{r}, t) \sim M^{*} / m R^{2}(t)$ at $|\mathbf{r}|<R(t)$ and $\tilde{n}=0$ at $|\mathbf{r}|>R(t)$. Eq.12 corresponds to the potential energy contribution to the action. As usual, the contribution from the kinetic energy is of the same order. Assuming that $R(t)=v_{F} t$ we get an estimate $S \sim\left(e M^{*}\right)^{2} d / m^{2} R_{W} v_{F}$. Thus, in principle, the wave function of the object is a coherent superposition of the wave functions of a uniform Fermi liquid and a Wigner crystal droplet. In this situation it is quite likely that the additional charge associated with such an object is not an integer. This is the reason why the nature of the ground state of the system remains unknown.

The quantum melting of the phases, which are intermediate between the bubble and the stripe phases is even more complicated and we leave this question for further investigation. 


\section{TRANSPORT PROPERTIES OF THE ELECTRON SYSTEM WITH DROPLETS OF A MINORITY PHASE EMBEDDED INTO THE MAJORITY ONE.}

In this section I will consider cases when quantum statistics of the system of droplets of the minority phase is not important.

The electron-electron scattering conserves the total momentum of the electron system and therefore does not contribute to the resistance of the system. To estimate it we have to consider the electron system in the presence of a random elastically scattering potential.

The electron transport picture in the electron liquid with partial phase separation is quite rich. In particular, there is a region of electron concentrations where the hydrodynamics of the electron liquid is similar to the hydrodynamics of the liquid crystals [26]. In this paper we consider only cases where either there are crystalline droplets of small concentration embedded in the liquid $\left(x_{L} \ll 1\right)$, or there are liquid droplets with $x_{W} \ll 1$ embedded into the crystal. In these situations, in principle, there are two types of current carriers in the system: electron quasiparticles and charged droplets of the minority phase. In this article we will ignore the contribution of the droplet motion to the charge transport. To illustrate the possible $T$ and $H$ dependence of the resistance we consider below only several limiting cases leaving a detailed analysis for future investigation.

\section{A. The case when crystal droplets of small concentration are embedded in the electron liquid.}

Let us consider the case $x_{W} \ll 1$ when crystalline droplets of small concentration are embedded into a Fermi liquid. We will assume here that the Wigner crystal droplets are either pinned by a small scattering potential, or have a short mean free path. We also assume that otherwise the impurities do not affect the thermodynamic properties of the system. The contribution to the resistance of the system from the scattering of quasiparticles on droplets has the form

$$
\rho=\frac{k_{F}}{e^{2} n l_{(e, W)}}
$$

where $k_{F}$ is the Fermi momentum of the Fermi liquid, $l_{(e, W)}=1 / N_{W} R_{W}$ is the quasiparticle mean free path, and $N_{W}=x_{W} / R_{W}^{2}$ is the concentration of droplets of the Wigner crystal. Thus as followes from Eq.1,2,9,13 at small $T$ the resistance of the electron system increases linearly in $T$. At small $H_{\|}$it increases quadratically in $H_{\|}$, while in the intermediate interval of $H_{\|}$it increases linearly in $H_{\|}$. The saturation of the magnetoresistance as a function of $H_{\|}$takes place at $H_{\|}>H_{\|}^{c}$ when the electron Fermi liquid gets polarized.

At $H_{\|}>H_{\|}^{c}$ the spin entropy of the Wigner crystal is frozen. Therefore, as it has been discussed, $x_{W}(T)$ and the resistance of the system do not have a significant $T$-dependence.

The $H_{\|}$dependence of the resistance $\rho\left(H_{\|}\right)$of the metallic phase at small $T$ is shown schematically in Fig.3a. The $T$ dependences of $\rho(T)$ at $H_{\|}=0$ and $H_{\|}>H_{\|}^{c}$ are shown in Fig.3b.

Eventually at high enough temperatures the crystalline droplets melt. Since at this point $r_{s} \gg 1$ the melting temperature $T_{m} \ll \Omega_{p}$ is much smaller than the plasma frequency at the wave vector of order of the inverse inter electron distance. Here $r_{S}$ is the ratio between the potential and the kinetic energies of electrons. Let us now discuss the $T$-dependence of $\rho(T)$ in this temperature interval. Though in this case the liquid is not degenerate, it is strongly correlated. Therefore the electron-electron scattering in the liquid is very effective and the local equilibrium is reached in a short time on a spatial scale of order $n^{-1 / 2}$. As a result, the flow of the electron liquid near an impurity can be considered in the framework of hydrodynamics. In the two-dimensional case the moving electron liquid exerts a force on an impurity, which is given by the Stokes formula $F \sim \eta u / \ln ((\eta / n u a)),[37]$. Here $u, \eta$ and $a$ are the liquid hydrodynamic velocity, viscosity of the electron liquid and the impurity radius respectively. In a system with a finite

concentration of impurities the logarithmic factor in the equation for $F$ should be substituted for $\ln \left(1 / a N_{i}^{1 / 2}\right)$, where $N_{i}$ is the concentration of impurities. Thus the resistance of the electron system has the form [39,38]

$$
\rho(T) \sim \frac{N_{i} \eta(T)}{e^{2} n^{2}} \ln ^{-1} \frac{1}{N_{i}^{1 / 2} a},
$$

The viscosity of the strongly correlated liquid in the semi-quantum regime has been considered theoretically in [40] for the case of liquid $\mathrm{He}^{3}$. It was conjectured that

$$
\eta \sim \frac{1}{T}
$$


We can apply this result to the case of electron liquid in the semi quantum regime $\left(T_{m} \ll T \ll \Omega_{p}\right)$ as well. Thus we arrive at the conclusion that at high temperatures the resistance should decrease inversely proportional to $T$ and that it should have a maximum at $T \sim T_{m} \sim E_{F}$. It is interesting to note that, as far as I know, the experimental data on the $T$-dependence of the viscosity of $\mathrm{He}^{3}$ in this relatively high temperature region are unavailable. However, we can look at data for the viscosity of $\mathrm{He}^{4}$, which in this temperature interval is supposed to be similar to $\mathrm{He}^{3}$ [40]. Though the experimental data for $\mathrm{He}^{4}$ are in a reasonable agreement with Eq.15, we would like to mention that the viscosity of $\mathrm{He}^{4}$ changes only by a factor of two in the temperature interval between $E_{F}$ and the evaporation point.

\section{B. Strongly correlated Fermi liquid in the presence of a scattering potential.}

Let us consider the case $n>n_{W}$, but $r_{s} \gg 1$. Then at $E_{F} \ll \Omega_{p}$ the electron system is a strongly correlated Fermi liquid. The main feature of such a liquid is that at small distances and at small (imaginary) times it behaves like a solid [34]. It has been suggested in [41] that the cross-section of quasiparticle scattering on a short ranged impurity with a radius of order $n^{-2}$, is significantly enhanced by the electron-electron interaction. The nature of the enhancement becomes especially clear if we consider the interval of electron densities close to the critical point $0<n-n_{L} \ll n_{L}$ and the case when the fluctuations of the external potential have a relatively small amplitude. Then the system can become split into the regions of a Fermi liquid and a Wigner crystal. I would like to mention that the linear $T$ and $H_{\|}$dependences of the resistance mentioned above are generic for strongly correlated electron system and are valid in this case as well.

The fractions of volume occupied by the Fermi liquid and the Wigner crystal depend on $n$ and therefore the system should exhibit a percolation-type zero-temperature metal-insulator transition as $n$ decreases and the area occupied by the Wigner crystal grows. There is however a significant difference with respect to the percolation transition, which originates from the fact that the position of the Wigner crystal-Fermi liquid boundary has quantum fluctuations. It is these fluctuations which determine the character of the electron transport near the transition point. The properties of the current carriers in this region are very different from properties of the Fermi liquid quasiparticles.

The significance of quantum fluctuations becomes even more clear if we consider quantum properties of the surface between the Wigner crystal and the Fermi liquid. At zero temperature the surface is a quantum object itself. There are at least two scenarios for the state of the surface at $T=0$ : it could be quantum smooth or quantum rough. In the first case the excitations of the surface are essentially the Ralaigh surface waves which conserve the charge inside the droplets. In the second case there is a new type of excitations at the surface: crystallization waves which do not conserve the total charge inside the droplets [42].

The problem of quantum roughening has been discussed in the framework of the properties of the boundary between solid and liquid $\mathrm{He}^{3}$ and $\mathrm{He}^{4}$ [42]. In the case of 2-d surfaces between a 3-d quantum liquid and crystal it has been argued that the surface is always quantum smooth [43]. At the moment nothing is known about the state of the boundary between 2-dimensional liquid and solid. We would like to mention, however, that quantum effects (including the quantum roughening) are more pronounced in the case considered above because it is two-dimensional and because the jump of the electron density in this case is small. In any case, due to quantum fluctuations, there is a region near the boundary whose properties are intermediate between the liquid and the solid properties.

In the conclusion of this section we would like to mention that the linear in $T$ increase of the resistance at small $T$ is a generic property of the model.

\section{A COMPARISON BETWEEN PREDICTIONS OF THE THEORY AND EXPERIMENTAL RESULTS IN SI MOSFET'S.}

\section{A. A review of experimental results on Si-MOSFET's.}

In this subsection we present a short list of experimental results on the high-mobility two-dimensional electron liquid in Si MOSFET's [1-13] which seem to be in contradiction with Fermi liquid theory and with the conventional single particle localization theory of disordered 2-dimensional conductors [14,15].

A. The electron system exhibits a "transition" as a function of $n$ from a metallic phase, where the resistance of the system saturates at low temperatures, to an insulating phase, where the resistance increases as $T$ decreases. The value of the critical concentration $n_{c}^{(M I)}$ of the transition depends on the amount of disorder in the sample and corresponds to $r_{s}=r_{s}^{c} \gg 1$. Here $r_{s}$ is the ratio between the electron potential and kinetic energies. 
B. At $T=0$ and for the electron concentration sufficiently close to the critical one, increasing the magnetic field $H_{\|}$parallel to the film drives the system toward the insulating phase [3,5,12]. Thus the critical metal-insulator concentration $n_{c}\left(H_{\|}\right)$increases with $H_{\|}$.

In the metallic phase $\left(n>n^{M I}\left(H_{\|}=0\right)\right)$ and at small $T$ the system exhibits a big positive magnetoresistance as a function of $H_{\|}$. This magnetoresistance saturates at $H_{\|} \geq H_{\|}^{c}(n)$ and $\rho\left(H_{\|}^{c}\right) / \rho(0) \gg 1[3,5]$.

C. In the metallic phase at $H_{\|}=0$ and $T<E_{F}$ the resistance $\rho(T)$ significantly increases with increasing temperature. The characteristic value of $d \ln \rho / d T>E_{F}^{-1}$ at small $T$ is large and depends on the value of $n-n_{c}$.

D. If at $H_{\|}>H_{\|}^{c}$ the system is still in the metallic phase $\left(n>n_{c}\left(H_{\|}\right)\right)$, the $T$-dependence of the resistance is much weaker than in the $H_{\|}=0$ case $[6,8,9]$.

E. The value of $H_{\|}^{c}$ decreases significantly as $n$ approaches $n_{c}^{M I}$.

\section{B. Qualitative explanation of experimental results.}

In this subsection we present a qualitative explanation of the experimental results [1-13]

A. The existence of the metal-insulator phase transition.

The theoretical picture presented above involves a transition between the liquid and the crystal as a function of $n$. Therefore it can explain qualitatively the existence of the metal-insulator transition observed in the experiments. Namely, the fractions of volume occupied by the Fermi liquid and the Wigner crystal depend on $n$ and therefore the system should exhibit a percolation-type zero-temperature metal-insulator transition as $n$ decreases and the area occupied by the Wigner crystal grows. The transition takes place when the Wigner crystal droplets overlap and block the electron transport through the Fermi liquid area.

The experimental values of $n_{c}^{(M I)}$ correspond to $r_{s} \sim 10-20$. At present it is difficult to say how close this value is to $n_{L}$, or $n_{c}$ in the pure case. The critical value for the transition $r_{s, c}=38$ [31] was obtained by numerical simulations. However it can not be applied to the case of electrons in Si MOSFET's because of the existence of two almost degenerate electron valleys. Another reason for possible inapplicability of the results of [31] to Si MOSFET's is that the calculations [31] were restricted to the case of zero temperature while the experiments have been performed at temperatures larger than the spin exchange energy in the Wigner crystal. Thus the Pomeranchuck effect has not been taken into account in [31]. Finally in the critical value of $r_{s}$ can be different in the disordered case.

B. The positive magnetoresistance of the metallic phase in the magnetic field parallel to the film.

The large positive magnetoresistance of the metallic phase in the parallel magnetic field is connected to the fact that $\chi_{W} \gg \chi_{L}$ and therefore the magnetic field parallel to the film drives the electron system toward the crystallization [34].(See Eqs.1,2,9,13). The magnetoresistance should saturate when $H_{\|}>H_{\|}^{c}$ and the electron Fermi liquid is polarized.

C. The temperature dependence of the resistance in the metallic phase.

The significant increase of the resistance as a function of temperature can be explained naturally as a consequence of the Pomeranchuk effect: The spin entropy of the Wigner crystal is larger than the entropy of the Fermi liquid and, therefore, the Wigner crystal regions grow with increasing temperature.

At high temperatures the droplets of crystal melt. It follows from Eqs.13,15 that in this temperature range the resistance decreases with increasing $T$. It is unclear at present whether the experiments support this picture.

D. The temperature dependence of the resistance in the metallic phase at large $H_{\|}$.

The Pomeranchuk effect disappears when $H_{\|}>H_{\|}^{c}$ and electron spins are fully polarized. In this case entropies of both the liquid and the solid are much smaller than the spin entropy of the crystal at $H_{\|}=0$. This means that in the leading approximation the areas occupied by the crystal and the liquid are $T$-independent. This explains the fact that in the metallic state at $H_{\|}>H_{\|}^{c}$ the $T$-dependence of the resistance is much smaller than in the case $H_{\|}=0$ [8,9]. (The ratio $d \rho / d T\left(H_{\|}=0\right) / d \rho / d T\left(H_{\|}>H_{\|}^{c}\right)$ can be as big as $\left.10^{2}\right)$.

E. The $n$-dependence of $H_{\|}^{c}$.

Perhaps the most direct check of the concept of the Fermi liquid which is close to crystallization is the measurement of the $n$-dependence of the magnetic field $H_{\|}^{c}(n)$ which polarizes the liquid. In the case of a non-interacting Fermi liquid $H_{\|}^{c}=H_{\|}^{c(0)}=E_{F} / \mu_{B}$ is a smooth function of $n$. The problem of the $n$ dependence of the critical magnetic field $H_{\|}^{c}(n)$ in strongly correlated liquids near the crystallization point and the origin of the strong enhancement of the spin susceptibility has been discussed in the context of the theory of liquid $\mathrm{He}^{3}$ [34]. It has been pointed out that there are two different scenarios for the origin of the significant (factor 15) enhancement of the spin susceptibility of $H e^{3}$ near the crystallization point. 
a)The system is nearly ferromagnetic which means that it is close to the Stoner instability. In this case the linear spin susceptibility $\chi_{L}$ is large, but other coefficients $a_{m}$ in the expansion of the energy

$$
\epsilon_{L}=\chi_{L}^{-1} M^{2}+a_{4} M^{4}+. . a_{m} M^{m}
$$

with respect to the spin magnetization $M$ are not small. Here $m$ is an even integer. In this case $H_{\|}^{c} \sim H_{\|}^{c(0)}$, which is relatively large.

b) The system is nearly solid. In this case both $\chi_{L}^{-1}$ and other coefficients $a_{m}$ in the Eq.15 decrease significantly as $n$ approaches the crystallization point $n_{c}$. In this case $H_{\|}^{c}(n) \ll H_{\|}^{c(0)}$ is small.

In the case of $\mathrm{He}^{3}$ the value of $H_{\|}^{c}(n)$ has never been measured. In the case of electrons in Si-MOSFET's it has been measured in [13]. A dramatical decrease of $H_{\|}^{c}(n)$ compared to $H_{\|}^{c(0)}$ has been observed as $n$ approaches $n_{c}$. In our opinion these experimental results support the model of a nearly solid Fermi liquid which is at $r_{s} \gg 1$. Conversely it is unlikely that the system is close to the Stoner instability.

\section{A comparison with alternative explanations of experiments on transport properties of the metallic phase of the electron system in Si MOSFET's.}

In this section we compare the explanation presented above with another explanation given in [44-49]. It is based on the fact that a single short range impurity in a metal creates Friedel oscillations of the electron density. Due to the electron-electron interaction the quasiparticles in the metal are scattered not only from the impurity but also from the modulations of the electron density. At finite temperature the Friedel oscillations decay exponentially at distances larger than the coherence length of the normal metal $v_{F} / T$. As a result, at low temperatures $(\rho(T)-\rho(0)) \sim C T$ with $C>0$ [44-47]. The exchange contribution to the resistance has not been taken into account in [44-47]. It has been shown [49] that in the presence of the exchange interaction at $\tau^{-1} \ll T \ll E_{F}$ the quantity $(\rho(T)-\rho(0))$ remains linear in $T$. However, at $r_{s} \ll 1$ the coefficient $C<0$ has negative sign, which is different from [44-47]. On the other hand, the expriments were performed in the regime $r_{s}>1$. They yeald a positive value of the coefficient $C>0$. At finite value of $r_{s} \sim 1$ the theory [49] predicts, that the coefficient $C$ chenges it's sign agein and become positive.

At relatively high temperatures $T \sim E_{F}$ one can neglect the interference corrections and the temperature dependence $\rho(T)$ is determined by the corresponding dependence of the thermal velocity and the electron scattering cross-section in a nondegenerate gas. In this regime $\rho(T)$ decreases with increasing $T$ [44,47]. At this point we would like to mention that at low $T$ Eqs.1,2,9,13 also predict the increase of the resistance linear in $T$ as well. Eqs.14,15 also predict the existence of the maximum of $\rho(T)$ at $T \sim E_{F}$ and decrease of the resistance at $T>E_{F}$. Thus, both the theory presented above and [44-49], in principle, could explain qualitatively the $T$-dependence of the resistance of the metallic state.

The situation with the magnetoresistance in the parallel magnetic field is more delicate. Strictly speaking, the interference corrections to the Drude conductivity calculated in [44-49] are relevant only at $r_{s} \ll 1$ and at small $T$ and $H_{\|}$, when the effects are small. On the other hand, at $H_{\|}>H_{\|}^{c}$, when the effects are large, the interference corrections are irrelevant, and the value of the magnetoresistance $\left(\rho\left(H_{\|}\right)-\rho(0)\right)$ is determined by the $H_{\|}$dependence of the Drude part of the resistance, which is due to the $H_{\|}$dependences of the Fermi momentum and the scattering cross-section of quasiparticles. (This part of the magneto resistance has not been taken into account in [49]). In this case a single electron theory yields a big and negative Drude magnetoresistance in contradiction with the experimental fact that it is big and positive.

In connection with this I would like to make several points.

a. At $r_{s}>1$, the diagrammatic calculations are not under control. In the framework of the conventional diagram technique it is difficult to account for all effects associated with the strong correlations at $r_{s} \gg 1$, including the giant renormalization of the electron scattering cross-section on impurities, the effects of phase separation, existence of the crystallization waves at the boundary between the two phases, and, finally, the Wigner crystallization itself.

b. The mechanism considered in [44-49] can not explain the increase of the resistance as a function of $H_{\|}$and $T$ which is significantly larger than unity. This is because the amplitude of the potential created by the Friedel oscillations of the density created by an impurity potential is smaller than the impurity potential itself. This theory also can not explain why the temperature dependence of the resistance $\rho(T)$ is suppressed so dramatically by the magnetic field parallel to the film. On the other hand, the theory presented in this article can explain these facts.

c. These mechanisms of $T$ and $H_{\|}$dependence of the resistance are based on very different physics. This can be seen, for example, from the fact that all single electron interference phenomena including the Friedel oscillations are smeared by finite temperature. Conversely, the fraction of the Wigner crystal increases with temperature. 
d. The amplitude of the Friedel oscillations is suppressed significantly in the case when the scattering potential is a smooth function of coordinates on the scale of the electron wavelength. This is exactly what happens when the scattering cross-section is significantly renormalized by the fact that near a short range impurity there are crystalline droplets and the position of the crystalline surface exhibits quantum fluctuations. Thus, in a sense, the mechanisms based on single electron interference and the mechanism based on quantum fluctuations of the solid-liquid boundary compete with each other.

In order to distinguish between these two mechanisms one needs to perform experiments on samples with higher mobility, where effects considered in this article will be much larger than unity.

Finally we would like to mention that the theory [44-49] may be relevant to experiments on the two dimensional electron system in GaAS samples [50,51].

\section{CONCLUSION}

We have shown that due to the existence of metallic gates in MOSFET's the phase separation is a generic property of pure electron liquids. The proof is based only on the assumption about the existence of the first order phase transition between the uniform Fermi liquid and the Wigner crystal phases and on electrostatic properties of two dimensional electron system. This distinguishes the theory presented above from the theories [52,38], which attempted to explain the experiments using the fact that in the 2-d electron liquid there is a first order phase transition between the Fermi-liquid and the Wigner crystal which is destroyed by small disorder. This difference, however, manifests itself only at relatively small values of $d$ and at relatively small amplitude of the disorder. Qualitative pictures of the $T$ and $H$-dependences of the resistance of the "metallic" phase are, roughly speaking, the same for the model presented above and for those considered in [38].

It is an open question how a disorder of finite amplitude affects the results presented above. In some regimes the system can demonstrate a glassy behavior characteristic for crystals in the presence of disorder. Experimental indications of glassy behavior of the electronic system in Si MOSFET's have been reported in [53,54].

In this paper we considered only bubble phases which exist near the critical concentrations $n_{L}$ and $n_{W}$. In the interval $n_{L}<n<n_{W}$ the system, will probably exhibit a sequence of quantum phase transitions. In particular, it is likely that at electron densities close to $n_{c}$ there is a stripe phase, which is similar to $[24,27,28]$.

In conclusion we would like to mention that the picture presented above is in many respects similar to the quantum critical point of strongly correlated electron systems considered in [55-57]. In particular, the Fermi liquid state with densities close to $n_{W}$ will demonstrate very large sensitivity to imperfections, which is characteristic for the "almost critical" quantum state [55].

This work was supported by Division of Material Sciences, U.S.National Science Foundation under Contract No. DMR-9970999. We would like to thank A.F. Andreev, E. Abrahams, S.Chakrovarty, A.Efros, M.Gershenson, S. Kivelson, S. Kravchenko, L. Levitov, D. Maslov, V. Pudalov, B. Shklovskii, M. Sarachik, and S. Vitkalov for useful discussions.

[1] S.V. Kravchenko, G.V. Kravchenko, J.E. Furneaux, V.M. Pudalov, M.D. Iorio, Phys. Rev. B. 50, 8039 (1994); S. Kravchenko, D. Simonian, M.P. Sarachik, W. Mason, J.E. Furneaux, Phys. Rev. Lett. 77, 4938, (1996); D. Popovic, A.B. Fowler, S. Washburn, Phys. Rev. Lett. 79, 1543 (1997).

[2] M.P. Sarachik, S.V. Kravchenko, Proc. Natl. Acad. Sci. USA 96, 5900 (1999); E. Abrahams, S.V. Kravchenko, M.P. Sarachik, Rev. Mod. Physics 73, 251 (2001).

[3] D. Simonian, S.V. Kravchenko, M.P. Sarachik, V.M. Pudalov, Phys. Rev. Lett. 79, 2304 (1997).

[4] S.V. Kravchenko D. Simonian, M.P. Sarachik, A.D. Kent, V.M. Pudalov, Phys. Rev. B. 58, 3553 (1998).

[5] V.M. Pudalov, G. Brunthaler, A. Prinz, G. Bauer, JETP Lett. 65, 932 (1997).

[6] V.M. Pudalov, G. Brunthaler, A. Prinz, G. Brauer, JETP Lett. 68, 442 (1998).

[7] V.M.Pudalov, M.D'Iorio, S.V.Kravchenko, J.W.Campbell, Phys.Rev.Lett. 70, 1866 (1993).

[8] K.M.Metres, H.Zeng, S.A.Vitkalov, M.P.Sarachik, T.M. Klapwijk, Phys.Rev.B, Rapid Communication, 63, R41101, (2000).

[9] A.A.Shashkin, S.V.Kravchenko, T.M.Klapwijk, Phys.Rev.Lett. 87, 266402 (2001)

[10] A.A.Shashkin, S.V.Kravchenko,V.T.Dolgopolov, T.M.Klapwijk, Pys.Rev.Lett. 87, 086801 (2001).

[11] T.Okamoto, K. Hosoya, S. Kawaji, and A. Yagi, Phys.Rev.Lett. 82, 3875, (1999).

[12] V. M. Pudalov, G. Brunthaler, A. Prinz, and G. Bauer, Phys.Rev.Lett. 88, 076401 (2002). 
[13] S. A. Vitkalov, Hairong Zheng, K. M. Mertes, M. P. Sarachik, T. M. Klapwijk, Phys.Rev.Lett., 87, 086401, (2001).

[14] E.A. Abrahams, P.W. Anderson, D.C. Licciardello, T.V. Ramakrishnan, Phys. Rev. Lett. 42, 673 (1979).

[15] L.P. Gorkov, A.I.Larkin, D.E. Khmelnitskii, Sov.Phys.JETP. Lett., 30, 228, (1979).

[16] A.I.Larkin, Sov.Phys.JETP 31, 784, 10070; A.I.Larkin, Yu.Ovchinnikov, J.L.T.Phys. 34, 409, (1979).

[17] A.F.Andreev, I.M. Lifshitz, Sov.Phys. JETP, 29 1107, (1969).

[18] T.Garel, S.Doniah, Phys.Rev. B, 26, 325, (1982).

[19] M.Seul, D.Andelman, Science, 267, 477, (1995).

[20] I.A. Nyrkiva, A.R.Khohlov, M.Dao, Macromolecules, 27, 4220, (1994).

[21] N.K. Glendenning, "Compact stars" Springer, 2000.

[22] A.Moreo, S,Yunoki, E,Dagatto, Science 283, 2034, (1999).

[23] M.Yu.Kagan, D.I.Khomskii, M.V.Mostovoi, Eur.Phys.J. B., 12, 217, (1999).

[24] V.Emery, S.Kivelson, Physica (Amsterdam) 209, 597, (1993).

[25] V.Emery, S.Kivelson, J. Tranquada, Proc. Natl. Sci. USA.

[26] Oganesyan V, Kivelson S. Fradkin E., Phys.Rev. B. 64, 195109, (2001).

[27] J.Zaanen, O.Gunnarson, Phys.Rev.B. 40, 7391, (1989).

[28] A.A. Koulakov, M.M. Fogler, B.I.Shklovskii, Phys.Rev.Lett. 76, 499, (1996); M.M.Fogler, A.A.Koulakov, B.I.Shklovskii, Phys.Rev. B 54, 1853, (1996).

[29] N.F. Mott "Metal-insulator transition", Taylor and Frenncis LTD, London, 1974.

[30] P.M. Chaikin, T.C.Lubensky, "Principles of condensed matter physics", Cambridge University Press, 1995.

[31] B. Tanatar, D.M. Ceperley, Phys. Rev. B 39, 5005 (1989).

[32] L.D.Landau, E.M.Lifshitz, Electrodynamics of Continuous Media, New York, Pergamon, 1984.

[33] S.Kivelson, S.Trugman, Phys.Rev. B. 33, 3629, (1986).

[34] B. Castaing, P. Nozieres, J. de Physique 40, 257 (1979).

[35] G. Benenti, X. Waintal and J.L. Pichard, Phys. Rev. Lett. 83, 1826 (1999).

[36] L. Levitov-S; A. Shytov, JETP.Lett. 66, 214. (1997).

[37] L.D. Landau, E.M. Lifshitz, Fluid Mechanics (Pergamon Press, 1984).

[38] B.Spivak, Phys.Rev.B. 64, 085317, (2001).

[39] M.Hruska, B.Spivak, Phys.Rev. B. 65, 033315, (2002).

[40] A.F. Andreev, Yu.A. Kosevich, Sov. Phys. JETP 50, 1218 (1979).

[41] B. Spivak, Sov. Phys. JETP 63, 760 (1986).

[42] A.F.Andreev, A.Yu. Parshin, Sov.Phys. JETP 48, 763, (1978).

[43] D.Fisher, J.Weeks, Phys.Rev.Lett. 50, 1077, (1983).

[44] F.Stern, S, Das Sarma, Solid State Electronics, 28, 158, (1985).

[45] T. Ando, A.B. Fowler, F. Stern, Rev.Mod.Phys. 54, 488, (1982).

[46] A.Gold, V.T.Dolgopolov, Phys.Rev.B, 33, 1076, (1986).

[47] S. Das Sarma and E.H. Hwang, Phys. Rev. Lett. 83, 164 (1999).

[48] V.T.Dolgopolov, A.Gold, JETP Letters, 71, 27, (2000).

[49] G.Zala, B.N. Narozhny, I.L.Aleiner, Phys. Rev. B 64, 214204, (2001); Phys.Rev.B. 65, 020201, (2001).

[50] J.Yoon, C.C.Li, D.Shahar, D.C. Tsui, M.Shayegan , Phys.Rev.Lett. 84, 4421, (2000);

[51] A.P.Mills, Jr., A.P.Ramires, L.N.Pfeiffer, K.W.West, Phys.Rew.Lett. 83, 2805, (1999).

[52] S.Chakrovarty, S.Kivelson, C.Nayak, K.Voelker, Phylos. Mag.79,859, (1999).

[53] V. M. Pudalov, M. E. Gershenson, H. Kojima, cond-mat/0201001.

[54] S. Bogdanovic, D. Popovic, cond-mat/0106545; J. Jaroszynski, Dragana Popovic, T. M. Klapwijk; cond-mat/0205226.

[55] R.B.Laughlin, G.G.Lonzarich, P.Monthoux, D.Pines; Adv. in Phys. 20, 361, (2001).

[56] S.Sachdev,Science, 288, 480, (2000).

[57] J. Ornstein, A. Millis, Science, 268, 468, (2000).

[58] J. Lorenzana, C. Castellani, C. Di Castro Phys. Rev. B 64, 235127 (2001). 


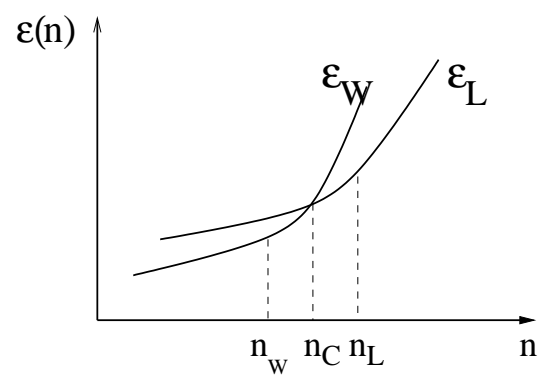

a)

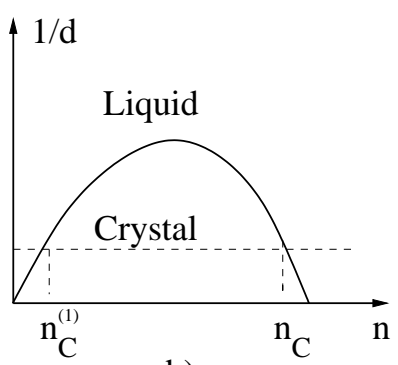

b)

FIG. 1. a. The dependence of the energy densities of the Wigner crystal and the Fermi liquid phases $\epsilon_{W, L}(n)$ on the electron density $n$. Symbols $W$ and $L$ correspond to the Wigner crystal and the Fermi liquid phases respectively. b. The effective phase diagram of the $2 \mathrm{D}$ electron system at zero temperature.

\begin{tabular}{|c|c|c|c|c|}
\hline WC & $\begin{array}{c}\text { Bubbles } \\
\text { of FL }\end{array}$ & $\begin{array}{l}\text { Stripe } \\
\text { phase }\end{array}$ & $\begin{array}{c}\text { Bubbles } \\
\text { of WC }\end{array}$ & FL \\
\hline
\end{tabular}

FIG. 2. The phase diagram of the $2 \mathrm{D}$ electron system at $T=0$. Symbols $W C$ and $F L$ correspond to the Wigner crystal and the Fermi liquid phases respectively. The shaded regions correspond to phases which are more complicated than the bubble and the stripe phases.

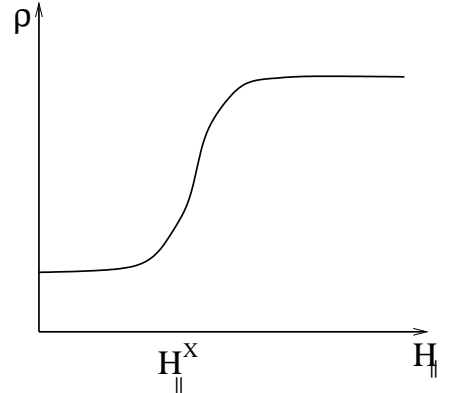

a)

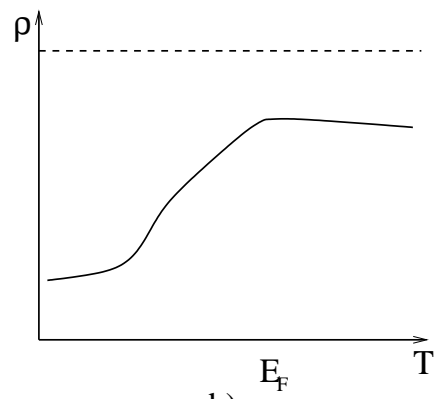

b)

FIG. 3. a)The $H_{\|}$dependence of the resistance $\rho\left(H_{\|}\right)$. b) The temperature dependence of the resistance $\rho(T)$. The solid line corresponds to the case $H_{\|}=0$, while the dashed line corresponds to the case $H_{\|}>H_{\|}^{c}$ 